\title{
At 68 years, unrecognised sleep apnoea is associated with elevated ambulatory blood
} pressure

\author{
Frédéric Roche*, Jean-Louis Pépin\#, Emilie Achour-Crawford*, Renaud Tamisier*, \\ Vincent Pichot*, Sébastien Celle*, Delphine Maudoux*, Florian Chouchou*, \\ Hourfil G. Ntougou-Assoumou*, Patrick Lévy\# and Jean Claude Barthélémy*, \\ on behalf of The PROOF Study Group
}

ABSTRACT: After the age of 65 yrs the specific impact of unrecognised sleep-related breathing disorders (SRBD) on 24-h blood pressure (BP) levels remains under debate. We tested the crosssectional relationship between the severity of obstructive sleep apnoea/hypopnoea (OSAH) and the increase of BP using ambulatory BP monitoring (ABPM) in the PROOF (PROgnostic indicator OF cardiovascular and cerebrovascular events study)-SYNAPSE (Autonomic Nervous System Activity, Aging and Sleep Apnea/Hypopnea study) cohort.

470 subjects (aged 68 yrs) neither treated for hypertension nor diagnosed for SRBD were included. All subjects underwent ABPM, and unattended at-home polygraphic studies. OSAH was defined by an apnoea/hypopnoea index $(\mathrm{AHI})>15 \cdot \mathrm{h}^{-1}$. The severity of the sleep apnoea was also quantified as the index of dips in oxyhaemoglobin saturation $>3 \%$ (ODI). Results are expressed in per protocol analysis.

Severe OSAH (AHI $>30 \cdot \mathrm{h}^{-1}, 17 \%$ of subjects) was associated with a significant $5 \mathrm{mmHg}$ increase in both diurnal and nocturnal systolic BP (SBP), and with a nocturnal $3 \mathrm{mmHg}$ increase in diastolic BP (DBP). Systolic (mean SBP $>135 \mathrm{mmHg}$ ) or diastolic (mean DBP $>80 \mathrm{mmHg}$ ) hypertension were more frequently encountered in subjects suffering from moderate (AHI 15-30) or severe OSAH. After adjustment, the independent association between severe OSAH and 24-h systolic hypertension remained significant (OR 2.42, 95\% $\mathrm{Cl} 1.1-5.4$ ). The relationship was further reinforced when SRBD severity was expressed using ODI $>10 \cdot \mathrm{h}^{-1}$.

The impact of unrecognised SRBD on BP levels also exists at the age of 68 yrs. The hypoxaemic load appears to be the pathophysiological cornerstone for such a relationship.

KEYWORDS: Ambulatory blood pressure, elderly, hypertension, obstructive sleep apnoea/ hypopnoea

bstructive sleep apnoea/hypopnoea (OSAH) syndrome is an independent risk factor for diurnal hypertension [1, 2] and has now been implicated as a risk factor for first or recurrent [3] strokes as well as post stroke mortality [4,5]. The amount of oxygen desaturation (hypoxaemic load) associated with apnoeas has been recognised as the pathophysiological cornerstone for the development of hypertension [6], via enhanced peripheral chemosensitivity [7], sympathetic over activity and of the early atherogenesis process associated with chronic intermittent hypoxia exposure [8]. However, sleep fragmentation or increased respiratory effort could also, by itself, precipitate sympathetically mediated hypertension [9].
Thus, sleep-related breathing disorders (SRBDs) have now been established as potential causes of secondary and resistant hypertension in middleage subjects [10]. In the elderly, the Sleep and Heart Health Study (SHHS) failed to demonstrate any significant association between SRBD and hypertension according to classical casual blood pressure (BP) measurements [11]. This lack of a relationship in the elderly is supported by the limited impact of OSAH syndrome on mortality in the elderly population [12] but can also be explained by the use of office BP measurement in the study.

The improved diagnostic and prognostic value of ambulatory BP monitoring (ABPM) compared with casual measurements has been firmly established
AFFILIATIONS

*Pole NOL et Service de Physiologie Clinique et de l'Exercice, CHU Nord EA 4607 SNA EPIS, Faculté de Médecine Jaques Lisfranc, USM, PRES Université de Lyon, Saint-

Etienne, and

"Pole Rééducation et Physiologie et Laboratoire HP2, INSERM U 1042 , Université Joseph Fourier, Grenoble, France.

CORRESPONDENCE

F. Roche

Service de Physiologie Clinique EFCR

CHU Nord - Niveau 6

F - 42055 Saint-Etienne

Cedex 2

France

E-mail: Frederic.Roche@univ-st-

etienne.fr

Received:

Oct 172010

Accepted after revision:

Jan 052012

First published online:

April 202012

European Respiratory Journal Print ISSN 0903-1936 Online ISSN 1399-3003 
[13-16]. From an epidemiological point of view, such methodology allows an improved and more objective evaluation for the influence of a medical or environmental stress on BP control. Using ABPM, several studies have already reported the high prevalence of previously ignored, and thus untreated, hypertension in patients addressed at the sleep laboratory for suspicion of OSAH syndrome. Nocturnal and diastolic hypertensions are the more frequently encountered abnormalities $[17,18]$.

Thus, on the one hand, SRBD remains undiagnosed and untreated in a large majority of patients [19], and it could, therefore, be postulated that a significant proportion of essential hypertension is related to the presence of occult OSAH [17]. On the other hand, the impact of unrecognised sleep apnoea on BP in a 24-h period has never been assessed in elderly subjects. This appears to be important as the ability to reduce BP is associated with a mortality and morbidity reduction even in elderly subjects [20]. Continuous positive airway pressure (CPAP) is the first line of treatment for OSAH. The impact of CPAP on controlling arterial BP is well documented in hypertensive as well as in normotensive OSAH patients, particularly during the night [21]. Therefore, this ventilator support can be used in the elderly [22] and could participate in the control of arterial BP within this population.

The aim of the present study was to evaluate, in a prospective cohort of homogenous elderly population, the impact of undiagnosed OSAH on BP levels by assessing unattended sleep apnoea or hypertension using a ventilatory polygraph and ABPM over a $24-h$ period.

\section{MATERIAL AND METHODS Population}

The present study sample was issued from the prospective observational PROOF (PROgnostic indicator OF cardiovascular and cerebrovascular events) study-SYNAPSE study (Autonomic Nervous System Activity, Aging and Sleep Apnea/Hypopnea) cohort, which consisted of 1,011 subjects all aged 65 yrs at inclusion in the PROOF study. The PROOF study was designed to assess the prognostic value of autonomic nervous system activity and its decline throughout the years and how this impacts on cardiovascular and cerebrovascular morbidity and mortality [23]. The PROOF population was selected from the electoral list of inhabitants in the town of Saint-Etienne (France) who were aged 65 yrs in 2001. Exclusion criteria were: previous myocardial infarction or stroke, heart failure, insulin-dependent diabetes mellitus, atrial fibrillation or anti-arrhythmic treatment, and a severe disease limiting life expectancy of $<5 \mathrm{yrs}$. All subjects were evaluated under standardised conditions, which are described in the protocol for the PROOF study [23]. Demographic characteristics included sex, age, smoking (past or current), body mass index $\left(\mathrm{BMI} \mathrm{kg} \cdot \mathrm{m}^{-2}\right)$, waist and hip circumference, alcohol intake, glycaemia and BP measurements. Daytime sleepiness was assessed with the Epworth Sleepiness Scale score. Both the PROOF and SYNAPSE studies were approved by the University Hospital and the Institutional Review Board/Independent Ethics Committee, (Consultative Committee for the Protection of Persons participating in Biomedical Research (Rhone-Alpes Loire, Saint-Etienne, France) and all subjects gave their written informed consent before participating.
854 subjects were re-evaluated 3 yrs after inclusion (aged 68 yrs). At this step, subjects of the cohort considered as normotensive according to the absence of self-reported history for hypertension and to the absence of prescription of antihypertensive drugs, were complementary assessed by an at-home respiratory polygraph sleep study (figure 1).

\section{Examination procedure}

The population study was examined in the Autonomic Nervous System consultation facilities at the University Hospital of SaintEtienne, (Saint-Etienne, France). Casual BP was systematically measured with a mercury sphygmomanometer at each visit, twice in a lying position after a 15-min rest period. Then one measurement was taken immediately after being in an upright position.

The use of antihypertensive drugs (angiotensin-converting enzyme inhibitors or angiotensin receptor blockers, beta-blockers, diuretics, calcium channel blockers, or others), history of hypertension and anthropometric data were assessed during a medical interview and examination.

24-h ambulatory BP levels were assessed using an ambulatory device recorder that used an auscultatory method (Diasys Integra, Novacor, Rueil-Malmaison, France), the measurements were programed every $15 \mathrm{~min}$ during the day and every $30 \mathrm{~min}$ during the night, with the cuff placed on the non-dominant arm. Average values of systolic BP (SBP) and diastolic BP (DBP) were calculated for the different recording periods: day, night and $24 \mathrm{~h}$. Hypertensive status was defined as $24 \mathrm{~h}$ ABPM $>135 /$ $80 \mathrm{mmHg}$, according to the recommended limits at the time the study was completed [24]. ABPM was performed the following day and after the at-home polygraphic monitoring so as to avoid discomfort or more awaken state due to automatic blood pressure measurements. A second ABPM was performed 1 week later $(n=28)$ when PB data were lacking for $\geqslant 30 \%$ of the

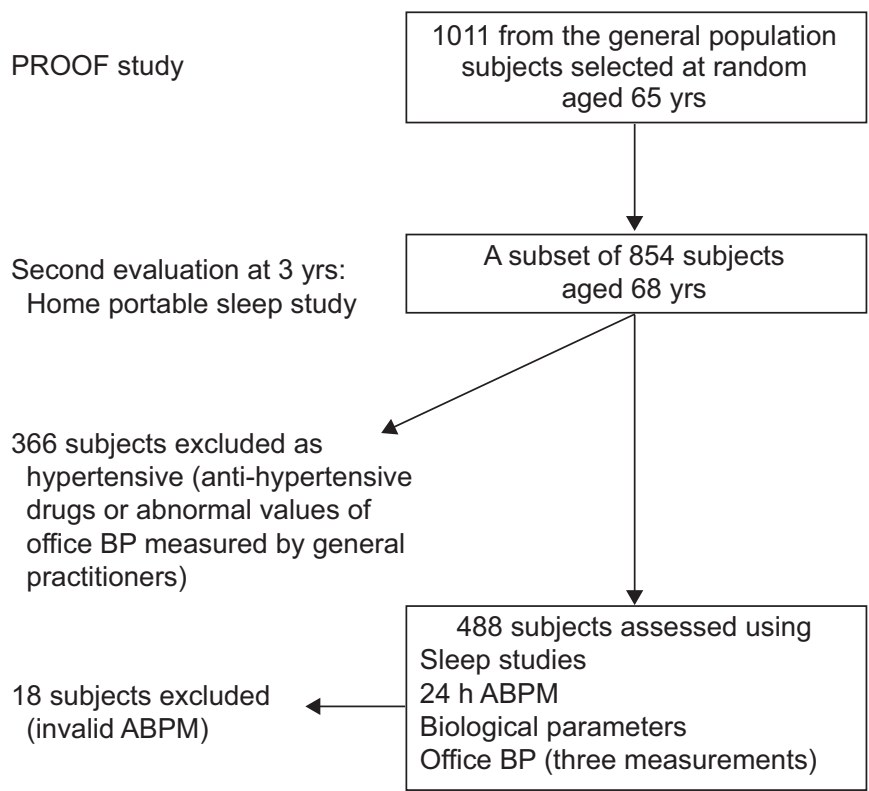

FIGURE 1. Flow chart depicting the study. PROOF: PROgnostic indicator OF cardiovascular and cerebrovascular events; BP: blood pressure; ABPM: ambulatory blood pressure monitoring 
BP measurements. At the end, ABPM was unavailable for technical problems in 18 subjects.

\section{At-home respiratory polygraphic sleep studies}

A ventilatory polygraph system (Hypno PTT, Tyco Healthcare, Puritan Bennett, Pleasanton, CA, USA) recorded ECG tracings (one lead), pulse oximetry (sampling rate $1 \mathrm{~Hz}$, minimal time duration to define an oxyhaemoglobin desaturation event: $10 \mathrm{~s}$ ), rib cage excursions (transthoracic impedance), body position and nasal pressure for measurement of ventilation. Nasal pressure is now recommended both for adult and paediatric populations as the noninvasive reference method for assessing airflow. Unattended studies in a patient's home are reliable for the present age group [25]. All the recordings were made overnight at home on the night following the in-hospital clinical examination, during which an experienced technician instructed all participants how to place the sensors. A second night of monitoring was performed when reported sleep latency exceeded $2 \mathrm{~h}$ on the first night $(n=5)$, or when one respiratory parameter was missing $(n=23)$. A recording duration of at least $5 \mathrm{~h}$ was required to validate the sleep study. All the recordings were validated visually and manually scored for apnoea/ hypopnoea events and inspiratory flow limitation episodes by investigators blinded to the other parameters with an intrascorer reliability of $88 \%$. To minimise potential over estimation of sleep duration, each subject completed a sleep diary to exclude from the analysis the wakefulness before lights off. Apnoea and hypopnoea events were defined according to previously published guidelines [26] and an oxyhaemoglobin desaturation threshold $>3 \%$ was chosen according to recommendations. Hypopnoea was defined as a $\geqslant 50 \%$ reduction in airflow from baseline value lasting a minimum of $10 \mathrm{~s}$ and apnoeas were defined as the absence of airway on the nasal cannula lasting for $\geqslant 10 \mathrm{~s}$. The apnoea/hypopnoea index (AHI; number of apnoea and/or hypopnoea events per hour of time in bed), as well as the oxyhaemoglobin desaturation index (ODI; number of desaturations per hour) were calculated. As indices of nocturnal hypoxaemia we also considered the mean nocturnal arterial oxygen saturation $\left(\mathrm{Sa}_{2} \mathrm{O}_{2}\right)$ the percentage of recording time spent with a $\mathrm{Sa}_{1} \mathrm{O}_{2}<90 \%$, and the minimal value recorded during sleep (nadir $\mathrm{Sa}_{\mathrm{a}} \mathrm{O}_{2}$ ).

Pulse transit time (PTT) was continuously measured as the interval between the ECG R-wave and the subsequent arrival of the pulse wave at the finger (usually the point on the pulse waveform that is $50 \%$ the height of the maximal value). PTT is $\sim 0.2-0.3 \mathrm{~s}$ when using the finger probe and is measured with an accuracy of $0.002 \mathrm{~s}$. PTT values are available with every heartbeat and are typically over sampled at $5 \mathrm{~Hz}$. Such an indicator of respiratory effort helps to qualify respiratory events as obstructive or central. The absence of rib cage movements associated with the absence of autonomic arousal defined a central respiratory event. Obstructive AHI and central AHI were calculated separately. Several groups have demonstrated, in both adults and children, that PTT is as accurate as oesophageal pressure for measuring respiratory effort both in frank and subtle respiratory events $[27,28]$.

According to previously reported data, an obstructive AHI $>15$ was considered in the elderly as the diagnostic threshold for obstructive sleep apnoea syndrome [29, 30]. Cases were defined as mild or moderate (AHI 15-30) or severe (AHI >30).
Increased hypoxaemic load was also defined as an ODI $>10 \cdot \mathrm{h}^{-1}$. Sleep study data were also presented according to interquartile comparisons.

\section{Statistical analysis}

The consistency of data was checked. When BP values or sleep study data deferred by two or more standards errors from the mean value, they were checked individually by the medical doctor in the original subject file. If the value was considered inconsistent, due to technical problems during the recordings, it was excluded from the analyses. Therefore, we expressed our results in per protocol and not in intention-to-treat analysis. Casual SBP, DBP and 24 ambulatory BP were compared between the OSAH states, using ANOVA with one between-factor. We used the Bonferroni method to adjust the level of significance for multiple comparisons. Analysis was adjusted for potential confounding factors when needed. Percentages of uncontrolled ambulatory BP (systolic, diastolic or systolo-diastolic hypertension) was calculated according to the severity of unrecognised OSAH and comparisons made using Pearson Chi-squared test.

To determine the independent association of hypertension and AHI, multiple logistic regressions analysis were built according to the presence of 24-h systolic hypertension, or 24-h diastolic hypertension. Hypertension and the severity of the OSAH (moderate or severe) relationship was assessed after adjustments for sex, BMI, presence of type 2 diabetes, dyslipidaemia, and smoking status. Complementary, adjusted logistic regression analysis was also performed according to the presence of 24-h systolic hypertension, or 24-h diastolic hypertension and the severity of ODI $\left(>10 \cdot h^{-1}\right)$.

Finally, using separate multiple logistic regression analysis, we compared the relationship of 24-h systolic or 24-h diastolic hypertensive status and $\mathrm{AHI}$ and ODI according to interquartile comparisons. A value of $\mathrm{p}<0.05$ was considered as statistically significant.

\section{RESULTS}

The flow chart of the study is depicted in figure 1. A total of 488 normotensive subjects presented with a validated polygraphic data. Among them, 18 showed invalidated ABPM unless a second ABPM had been performed 1 week later. The final dataset included a total of 470 subjects. Clinical, anthropometric and sleep study data are shown in table 1 according to the presence and the severity of OSAH. The subset of elderly $(n=18)$ excluded from the analysis demonstrated anthropometric, sleep study data (table 1) as well as casual SBP and DBP values similar to the final $(n=470)$ dataset (SBP and DBP were $136.3 \pm 15.6$ versus $135.2 \pm 15.7 \mathrm{mmHg}$ and $84.5 \pm 8.1$ versus $84.2 \pm 8.5 \mathrm{mmHg}$, respectively; not significant).

In comparison to subjects without apnoeic, severe OSAH (AHI $>30 \cdot \mathrm{h}^{-1}, 17 \%$ of included subjects) was associated with a significant increase of $\geqslant 5 \mathrm{mmHg}$ in both diurnal and nocturnal ambulatory SBP and with a nocturnal increase of $3 \mathrm{mmHg}$ in ambulatory DBP (table 2). Presence of a moderate OSAH was associated with an elevation of $3 \mathrm{~mm} \mathrm{Hg}$ of SBP during daytime and an increase of $5 \mathrm{~mm} \mathrm{Hg}$ of SBP during the night period. A limited, but significant, increase of DBP was also observed during the night period in this population of moderate OSAH. Interestingly, casual BP measurements did not differ according 
TABLE 1 Clinical and sleep study data

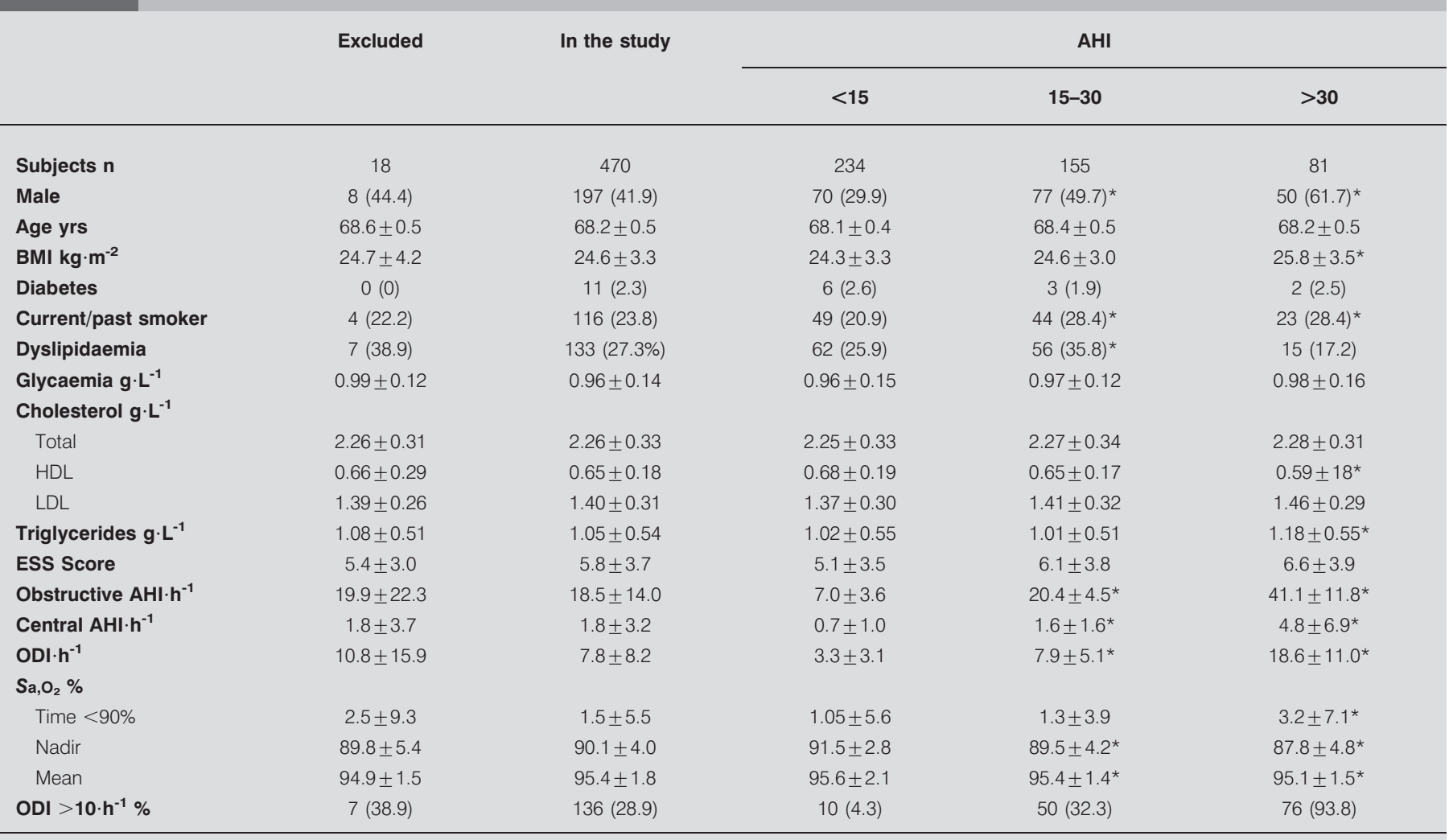

Data are presented as $n, n(\%)$ or mean \pm SE. AHI: apnoea/hypopnoea index; BMI: body mass index; HDL: high density lipoprotein; LDL: Iow-density lipoprotein; ESS: Epworth Sleepiness Scale; ODI: oxyhaemoglobin desaturation index; $\mathrm{Sa}_{2} \mathrm{O}_{2}$; arterial oxygen saturation. *: $\mathrm{p}<0.05$ from subjects presenting with an $\mathrm{AHI}<1$.

to the presence or not of an unrecognised SRBD and sexstratified analysis confirmed significant relationships between $\mathrm{AHI}$ and BP levels in males and females (table 2).

Table 3 is summarising the respective rates of hypertension as defined by ABPM in nonapnoeic, moderate and severe OSAH subjects. Abnormal systolic (mean 24-h ambulatory $\mathrm{BP}>135 \mathrm{mmHg}$ ) or diastolic (mean 24-h ambulatory BP $>80$ $\mathrm{mmHg}$ ) hypertension was more frequently encountered in subjects suffering from moderate or severe OSAH ( $p<0.02$ and $\mathrm{p}<0.03$, respectively) in comparison to nonapnoeic patients. According to the presence of a moderate and a severe OSAH, the total prevalence of uncontrolled systolic or diastolic hypertension reaches $22.6 \%$ and $29.6 \%$, respectively.

Multiple logistic regression analysis showed, after adjustment on age, gender, BMI, diabetes, dyslipidaemia, smoking status, and alcohol consumption, an independent association between severe OSAH (AHI >30) and 24-h systolic hypertension (OR $2.42,95 \%$ CI 1.1-5.4) but not with moderate OSAH (AHI 1530). The increased risk of systolic hypertension and of diastolic hypertension (24-h ABPM) was more pronounced when SRBD was expressed using an ODI $>10 \cdot \mathrm{h}^{-1}$ (OR 2.43, 95\% CI 1.45-4.1 for 24-h systolic hypertension and OR $2.52,95 \%$ CI $1.45-4.36$ for 24-h diastolic hypertension).

Separate multiple logistic regression analysis were made using successive ODI and AHI. The SRBD severity is presented according to interquartile comparisons for ODI parameters. The analysis confirmed the significant relationship between OSAH and silent hypertension for SBP as well as for DBP (table 4). The relationship was found to be significant for AHI parameter and silent systolic hypertension only.

\section{DISCUSSION}

Our results showed that, in an elderly population, unrecognised severe sleep apnoea is associated with a diurnal and nocturnal elevation of $\mathrm{BP} \geqslant 5 \mathrm{mmHg}$. The link between sleep apnoea and elevated BP remained significant even after adjustment for age, sex, BMI, diabetes, dyslipidaemia, smoking status, and alcohol consumption. Hypertension as defined by ABPM measurements reached a prevalence of nearly $30 \%$ in the elderly subjects with unrecognised OSAH syndrome. The ODI $>10 \cdot \mathrm{h}^{-1}$ was the parameter demonstrating the stronger association with increases in BP.

Hypertension in humans is a very common condition affecting over 1 billion people worldwide [31]. The cause of hypertension is known for $\sim 5 \%$ of cases, the other $95 \%$ cases are classified as "essential hypertension". OSAH syndrome is an independent risk factor for office hypertension [2, 10]. The Wisconsin Sleep Cohort Study [1] prospectively investigated in middle aged subjects the association between OSAH severity and incident hypertension at 4 yrs. Subjects with an AHI $>15$ events per hour at initial evaluation had a threefold risk ratio for developing hypertension over the 4-yr follow-up compared 


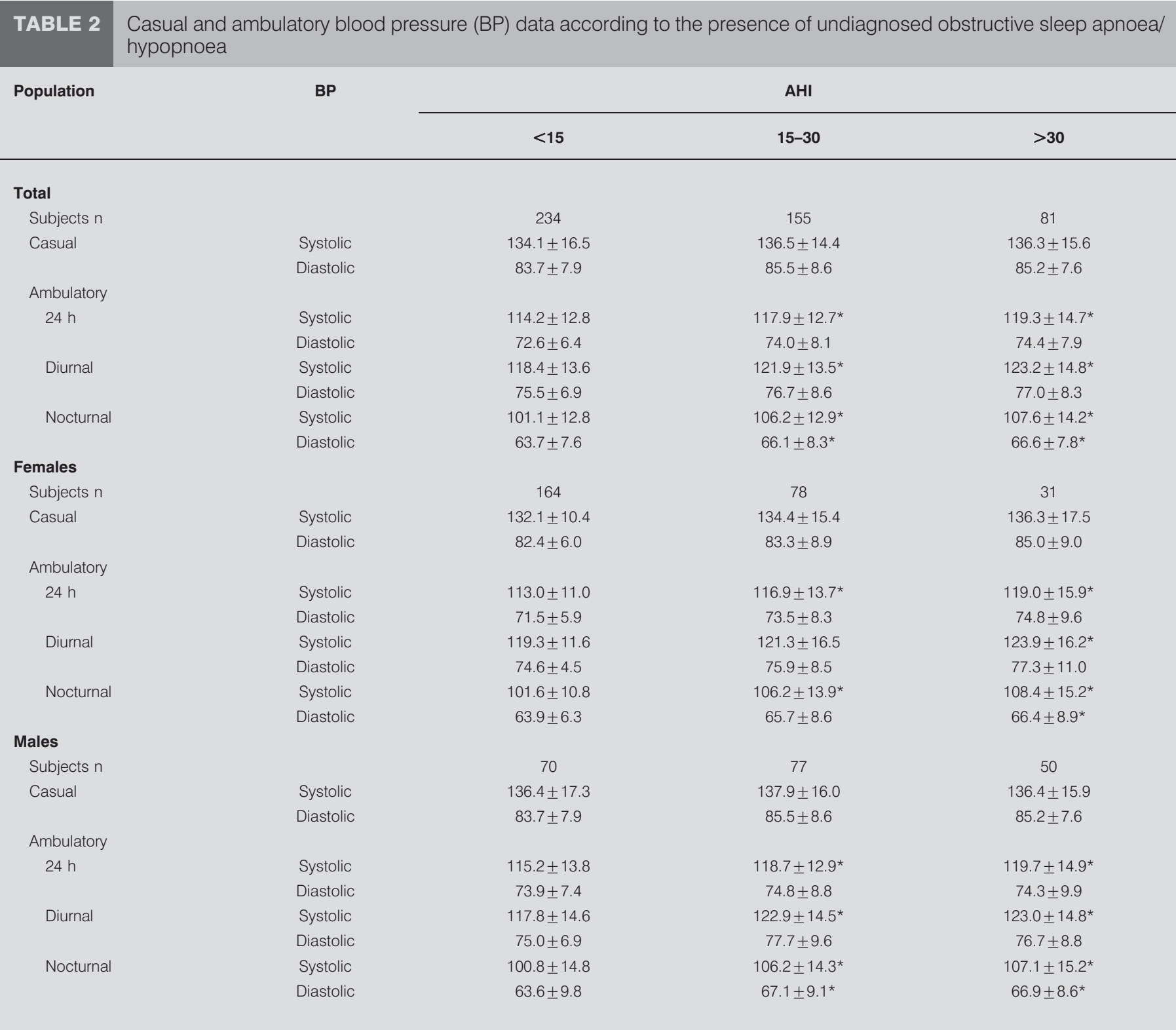

AHI: apnoea/hypopnoea index. An adjustment was made for age, body mass index dyslipidaemia, type 2 diabetes, smoking status, and alcohol consumption. *: p<0.05 from subjects presenting an $\mathrm{AHI}<15$.

TABLE 3 Contingency tables testing the association between unrecognised obstructive sleep apnoea and rate of systolic or diastolic hypertension as defined by ambulatory blood pressure monitoring

\begin{tabular}{|c|c|c|c|c|}
\hline & $<15$ & $15-30$ & $>30$ & All \\
\hline Subjects $n$ & 234 & 155 & 81 & 470 \\
\hline Systolic hypertension & $16(6.8)$ & $16(10.3)^{*}$ & $15(18.5)^{\star}$ & $47(10.0)$ \\
\hline Diastolic hypertension & $30(12.8)$ & $29(18.7)^{\star}$ & $19(23.5)^{\star}$ & $78(16.6)$ \\
\hline
\end{tabular}

Data presented as $n(\%)$. AHI: apnoea/hypopnea index *: $p<0.05$. 


\begin{tabular}{|c|c|c|c|c|c|c|c|c|}
\hline TABLE 4 & $\begin{array}{l}\text { comp } \\
\text { d brea } \\
\text { dex ( }\end{array}$ & for ti & $\begin{array}{l}\text { d of th } \\
\text { Sever }\end{array}$ & $\begin{array}{l}\text { equel } \\
\text { s defir }\end{array}$ & $\begin{array}{l}\text { f syst } \\
\text { using }\end{array}$ & $\begin{array}{l}\text { id dias } \\
\text { emogl }\end{array}$ & $\begin{array}{l}\text { ertension accor } \\
\text { turation index }\end{array}$ & $\begin{array}{l}\text { degree of } \\
\text { apnoea/ }\end{array}$ \\
\hline Hypertension & Index & Q1 & Q2 & Q3 & Q4 & $\mathbf{R}$ & OR (95\% Cl) & p-value \\
\hline \multirow[t]{2}{*}{ Diastolic } & ODI & 10.6 & 12.2 & 15.7 & 33.7 & 0.178 & $4.31(2.08-8.92)$ & 0.0003 \\
\hline & $\mathrm{AHI}$ & 11.3 & 13.6 & 21.1 & 22.3 & 0.050 & $1.83(0.93-3.59)$ & 0.08 \\
\hline
\end{tabular}

to those without any apnoea. In accordance with these results, it is now recommended by both European and American guidelines to seek sleep apnoea as an explanatory factor for hypertension $[19,32]$.

The risk of hypertension in OSAS patients may be particularly pronounced in younger adult patients ( $<50 \mathrm{yrs}$ ) compared with older ones [33]. The SHHS study failed to find a significant association between SRBD and systolic/diastolic hypertension in subsets of elderly subjects [11]. In the SHHS study, analyses excluding participants treated for hypertension ( $49 \%$ of the population aged $\geqslant 60 \mathrm{yrs}$ ) did not change the results. These results were in accordance with mortality studies of sleep apnoea patients showing maximum risk of dying in younger patients and a pronounced age decline in relative mortality reaching nonsignificant levels in patients aged $\geqslant 50$ yrs [12]. A recent hypothesis is a pre-conditioning effect [34] due to repeated intermittent hypoxia that may promote some degree of cardiovascular protection and a reduced risk of acute coronary heart disease, owing to the development of coronary collaterals. Another recent experimental study in rats exposed to chronic intermittent hypoxia suggests an age-related resistance to oxidative stress in the myocardium [35]. The sympathetic burst in response to intermittent hypoxic events could be reduced and this could explain the relatively small secondary increases in BP.

Actually, the major difference between the SHHS study and the PROOF-SYNAPSE studies were BP measurements methods for normotensive/hypertensive status determination. ABPM has demonstrated its ability to detect subjects with normal clinic but high ambulatory BP (masked hypertension) and also to determine white-coat hypertension. The prevalence rate of masked hypertension in newly diagnosed middle-aged OSAH patients who had been referred to a Sleep Laboratory has been founded to be up to $30 \%$ by several authors $[17,18]$. To our best knowledge, the current study is the first one to evaluate, in an elderly population, masked hypertension and its relationship with occult OSAH. According to our results, masked hypertension is also frequently associated to occult SRBD in elderly people, particularly in subjects suffering with $\mathrm{AHI}>30 \cdot \mathrm{h}^{-1}$ or ODI $>10 \cdot h^{-1}$. This dose-response relationship between OSAH syndrome severity and hypertension was found more strictly for systolic hypertension than for diastolic hypertension.
While moderate, such increases in BP levels could have a direct or an indirect impact in atherogenesis development. Recent studies have shown abnormal vascular responses in patients with OSAH syndrome even in patient without any over cardiovascular disease, and are similar to those occurring in patients with essential hypertension $[36,37]$. OSAH may be associated with increased vasoconstrictor sensitivity [38], impaired endotheliumdependent vasodilatation to acetylcholine and bradykinin [39, 40], and decreased $\alpha$ - and $\beta 2$-adrenergic vascular responses [41]. It has been shown that endothelial dysfunction, one of the factors underlying cardiovascular morbidity in SRBD, is clearly present in elderly OSAH patients and appears related to hypoxaemia severity indices [42]. The high prevalence of masked hypertension that we have demonstrated in this study might be one of the underlying mechanisms.

The treatment of symptomatic OSA syndrome with CPAP clearly attenuates the cardiovascular consequences of this disease and reduces BP levels in both hypertensive and normotensive patients with OSAH syndrome [42, 43], irrespective of sleepiness [44]. Such beneficial antihypertensive therapeutic effect has been most marked in those with the highest index of oxyhaemoglobin desaturation/resaturations during sleep associated to the most frequent apnoeas and hypopnoeas that occur. Elderly symptomatic SRBD patients tolerate CPAP as well as younger patients and should be effectively treated. The observance rate of nocturnal CPAP of an elderly asymptomatic or paucisymptomatic population would be expected to be low [39].

Our study suffers limitations. Our cohort is homogeneous in age and involves a low risk population, since none of the subjects presented myocardial infarction, stroke, heart failure, or atrial fibrillation at the time of inclusion. The performed a per protocol analysis. Since failure of ABPM is sometimes associated with severe hypertension or high variability of $\mathrm{BP}$, it could be speculated that subjects excluded from the protocol had a higher SBP or DBP than the group under study. Actually, it was not the case. The clinical characteristics of the excluded subset $(n=18)$, as well as their casual BP measurements, were not statistically different from the 470 elderly subjects who took part in the study. The subjects excluded from the present study had undergone ABPM 20 months previously. At that time, their blood pressure measurements were not different 
from those of the 470 subjects included in the final study group. (24-h SBP was $116.3 \pm 15.4 \mathrm{mmHg}$ versus $114.7 \pm 10.8 \mathrm{mmHg}$, respectively, and 24-h DBP was $75.1 \pm 8.3$ versus $74.8 \pm 6.8 \mathrm{mmHg}$; p-value significant). We do not think that an intention-to-treat analysis would have led to any different conclusions. The results presented in this study are only cross sectional data and the percentage of masked hypertensive subjects is relatively high in our population. To avoid altering sleep stability by repeated BP measurements during night we undertook ABPM procedures and sleep studies separately. This is a survival cohort study and the clinical impact of SRBD on hypertensive status as well as the independent effect of silent OSAH on cardiovascular and cerebrovascular morbidity and/or mortality should be evaluated later. Some selection bias could account for our findings. First of all, despite the wide spectrum of SRBD present in our population, mild cases were prevalent, the absence of severe cases reducing the relationship between $\mathrm{DBP}$ and $\mathrm{AHI}$ especially in females. However, this finding is common in clinical and epidemiological studies, severe cases being more common in young patients. Another factor is that the examined healthy elderly subjects had not been institutionalised and, therefore, they may constitute a survivor group more resistant to vascular risk. This latter possibility could be suggested by the different clinical aspects of SRBD in the elderly, in which neither sleepiness nor common predisposing factors appear strongly related to apnoea density.

In conclusion, unrecognised SRBD enhanced nocturnal as well as diurnal BP in 68-yr-old subjects. The frequency of hypoxaemia/ reoxygenation cycles appears to be the pathophysiological cornerstone of such a relationship in the present cross sectional analysis. The impact of such findings on subsequent cardiovascular and cerebrovascular morbidity is still questionable.

\section{SUPPORT STATEMENT}

This study was supported by a grant from the French Ministery of Health (Cellule Projet Hospitalier de Recherche Clinique National, Direction de la Recherche Clinique, CHU Saint-Etienne; Appel d'Offre 1998 and Appel d'Offre 2002), and by a grant from the "L'Association de Recherche SYNAPSE" (President M. Segura).

\section{CLINICAL TRIAL}

This study is registered at ClinicalTrials.gov with clinical trial identifier numbers NCT00759304 and NCT00766584.

\section{STATEMENT OF INTEREST}

None declared.

\section{REFERENCES}

1 Peppard PE, Young T, Palta M, et al. Prospective study of the association between sleep-disordered breathing and hypertension. N Engl J Med 2000; 342: 1378-1384.

2 Nieto FJ, Young TB, Lind BK, et al. Association of sleep-disordered breathing, sleep apnea, and hypertension in a large communitybased study. JAMA 2000; 283: 1829-1836.

3 Valham F, Mooe T, Rabben T, et al. Increased risk of stroke in patients with coronary artery disease and sleep apnea: a 10-year follow-up. Circulation 2008; 118: 955-960.

4 Marin JM, Carrizo SJ, Vicente E, et al. Long-term cardiovascular outcomes in men with obstructive sleep apnoea-hypopnoea with or without treatment with continuous positive airway pressure: an observational study. Lancet 2005; 365: 1046-1053.
5 Sahlin C, Sandberg O, Gustafson Y, et al. Obstructive sleep apnea is a risk factor for death in patients with stroke: a 10-year followup. Arch Intern Med 2008; 168: 297-301.

6 Norman D, Loredo JS, Nelesen RA, et al. Effects of continuous positive airway pressure versus supplemental oxygen on 24-hour ambulatory blood pressure. Hypertension 2006; 47: 840-845.

7 Fletcher EC. Sympathetic over activity in the etiology of hypertension of obstructive sleep apnea. Sleep 2003; 26: 15-19.

8 Imadojemu VA, Mawji Z, Kunselman A, et al. Sympathetic chemoreflex responses in obstructive sleep apnea and effects of continuous positive airway pressure therapy. Chest 2007; 131: 1406-1413.

9 Dematteis M, Julien C, Guillermet C, et al. Intermittent hypoxia induces early functional cardiovascular remodeling in mice. Am J Respir Crit Care Med 2008; 177: 227-235.

10 Calhoun DA, Jones D, Textor S, et al. Resistant hypertension: diagnosis, evaluation, and treatment. A Scientific Statement from the American Heart Association Professional Education Committee of the Council for High Blood Pressure Research. Circulation 2008; 117: e510-526.

11 Haas DC, Foster GL, Nieto FJ, et al. Age-dependent associations between sleep-disordered breathing and hypertension: importance of discriminating between systolic/diastolic hypertension and isolated systolic hypertension in the Sleep Heart Health Study. Circulation 2005; 111: 614-621.

12 Lavie P, Lavie L, Herer P. All-cause mortality in males with sleep apnoea syndrome: declining mortality rates with age. Eur Respir J 2005; 25: 514-520.

13 Staessen JA, Asmar R, De Buyzere M, et al. Task Force II: blood pressure measurement and cardiovascular outcome. Blood Press Monit 2001; 6: 355-370.

14 Dolan E, Stanton A, Thijs L, et al. Superiority of ambulatory over clinic blood pressure measurement in predicting mortality: the Dublin Outcome Study. Hypertension 2005; 46: 156-161.

15 Clement DL, De Buyzere ML, De Bacquer DA, et al. Prognostic value of ambulatory blood pressure recordings in patients with treated hypertension. N Engl J Med 2003; 348: 2407-2415.

16 Parati G, Pickering TG. Home blood-pressure monitoring: US and European consensus. Lancet 2009; 373: 876-878.

17 Baguet JP, Lévy P, Barone-Rochette G, et al. Masked hypertension in obstructive sleep apnea syndrome. J Hypertens 2008; 26: 885-892.

18 Grote L, Hedner J, Peter JH. Mean blood pressure, pulse pressure and grade of hypertension in untreated hypertensive patients with sleep-related breathing disorder. J Hypertens 2001; 19: 683-690.

19 Somers VK, White DP, Amin R, et al. AHA/ACCF Scientific Statement. Sleep apnea and cardiovascular disease. Circulation 2008; 118: 1080-1111.

20 Beckett NS, Peters R, Fletcher AE, et al. Treatment of hypertension in patients 80 years of age or older. N Engl J Med 2008; 358: 1887-1898.

21 Becker HF, Jerrentrup A, Ploch T, et al. Effect of nasal continuous positive airway pressure treatment on blood pressure in patients with obstructive sleep apnea. Circulation 2003; 107: 68-73.

22 Launois SH, Pépin JL, Lévy P. Sleep apnea in the elderly: a specific entity? Sleep Med Rev 2007; 11: 87-97.

23 Barthélémy JC, Pichot V, Dauphinot V, et al. Autonomic nervous system activity and decline as prognostic indicators of cardiovascular and cerebrovascular events. The "PROOF" Study. Neuroepidemiology 2007; 29: 18-28.

24 Pickering TG, Hall JE, Appel LJ, et al. Recommendations for blood pressure measurement in humans and experimental animals. Part 1: Blood pressure measurement in humans: a statement for professionals from the Subcommittee of Professional and Public Education of the American Heart Association Council on High Blood Pressure Research. Circulation 2005; 111: 697-716.

25 Ancoli-Israel S, Kripke DF, Mason W, et al. Comparisons of home sleep recordings and polysomnograms in older adults with sleep disorders. Sleep 1981; 4: 283-291. 
26 Sleep-related breathing disorders in adults: recommendations for syndrome definition and measurement techniques in clinical research. The report of an American Academy of Sleep Medicine Task Force. Sleep 1999; 22: 667-689.

27 Pépin JL, Delavie N, Pin I, et al. Pulse transit time improves detection of sleep respiratory events and microarousals in children. Chest 2005; 127: 722-730.

28 Argod J, Pépin JL, Smith RP, et al. Comparison of esophageal pressure with pulse transit time as a measure of respiratory effort scoring obstructive nonapneic respiratory events. Am J Respir Crit Care Med 2000; 162: 87-93.

29 Mant A, Saunders NA, Eyland EA. Sleep habits and sleep related respiratory disturbance in an older population. In: Horne J, Lovie P, eds. Sleep '88. Stuttgart, Gustav Fischer Verlag, 1989; pp 260-261.

30 American Academy of Sleep Medicine. International Classification of Sleep Disorders, Diagnostic and Coding Manual. 2nd Edn. Westchester, American Academy of Sleep Medicine 2005

31 World Health Organization. Global Health Observatory. www. who.int/gho/ncd/risk_factors/blood_pressure_prevalence/en Date last updated: January, 2012. Date last accessed: July 16, 2012.

32 Mancia G, De Backer G, Dominiczak A, et al. 2007 ESH-ESC Guidelines for the management of arterial hypertension: the task force for the management of arterial hypertension of the European Society of Hypertension (ESH) and of the European Society of Cardiology (ESC). Blood Press 2007; 16: 135-232.

33 Grote L, Ploch T, Heitmann J, et al. Sleep-related breathing disorder is an independent risk factor for systemic hypertension. Am J Respir Crit Care Med 1999; 160: 1875-1882.

34 Lavie L, Lavie P. Ischemic preconditioning as a possible explanation for the age decline relative mortality in sleep apnea. Med Hypotheses 2006; 66: 1069-1073.

35 Bianchi G, Di Giulio C, Rapino C, et al. p53 and p66 proteins compete for hypoxia-inducible factor 1 alpha stabilization in young and old rat hearts exposed to intermittent hypoxia. Gerontology 2006; 52: 17-23.
36 Kraiczi H, Peker Y, Caidahl K, et al. Blood pressure, cardiac structure and severity of obstructive sleep apnea in a sleep clinic population. J Hypertens 2001; 19: 2071-2078.

37 Kraiczi H, Caidahl K, Samuelsson A, et al. Impairment of vascular endothelial function and left ventricular filling: association with the severity of apnea-induced hypoxemia during sleep. Chest 2001; 119: 1085-1091.

38 Kraiczi H, Hedner J, Peker $\mathrm{Y}$, et al. Increased vasoconstrictor sensitivity in obstructive sleep apnea. J Appl Physiol 2000; 89: 493-498.

39 Kato M, Roberts-Thomson P, Phillips BG, et al. Impairment of endothelium-dependent vasodilation of resistance vessels in patients with obstructive sleep apnea. Circulation 2000; 102: 2607-2610.

40 Duchna HW, Guilleminault C, Stoohs RA, et al. Vascular reactivity in obstructive sleep apnea syndrome. Am J Respir Crit Care Med 2000; 161: 187-191.

41 Grote L, Kraiczi H, Hedner J. Reduced $\alpha$ - and $\beta_{2}$-adrenergic vascular response in patients with obstructive sleep apnea. Am J Respir Crit Care Med 2000; 162: 1480-1487.

42 Nieto FJ, Herrington DM, Redline S, et al. Sleep apnea and markers of vascular endothelial function in a large community sample of older adults. Am J Respir Crit Care Med 2004; 169: 354-360.

43 Faccenda JF, MacKay TW, Boon NA, et al. Randomized placebocontrolled trial of continuous positive airway pressure on blood pressure in the sleep apnea-hypopnea syndrome. Am J Respir Crit Care Med 2001; 163: 344-348.

44 Pepperell JC, Ramdassingh-Dow S, Crosthwaite N, et al. Ambulatory blood pressure after therapeutic and subtherapeutic nasal continuous positive airway pressure for obstructive sleep apnoea: a randomised parallel trial. Lancet 2002; 359: 204-210.

45 Hui DS, To KW, Ko FW, et al. Nasal CPAP reduces systemic blood pressure in patients with obstructive sleep apnoea and mild sleepiness. Thorax 2006; 6: 1083-1090. 